\title{
Failed Breast Feeding among Egyptian Women at One Month Postpartum: A Cross-Sectional Community Based Study
}

\author{
Ranya Aly Hegazy ${ }^{1^{*}}$, Shaimaa Baher Abdelaziz ${ }^{2}$, Ahmed Abdelkader Fahmy ${ }^{3}$ and Eman Kamal Shaeer ${ }^{4}$ \\ ${ }^{1}$ Department of Pediatrics, Faculty of Medicine, Cairo University, Giza, Egypt \\ ${ }^{2}$ Department of Community and Public Health, Faculty of Medicine Cairo University, Giza, Egypt \\ ${ }^{3}$ Department of Obstetrics and Gynecology, Faculty of Medicine, Ain Shams University, Eman Kamal Shaeer, Giza, Egypt \\ ${ }^{4}$ Department of Obstetrics and Gynecology, Faculty of Medicine, Cairo University, Giza, Egypt
}

"Corresponding author: Ranya Aly Hegazy, Department of Pediatrics, Faculty of Medicine, Cairo University 3 Ramez st. off Shehab st Mohandeseen, Giza 12316, Egypt, Tel: +241-06-267; E-mail: r_hegazy@hotmail.com

Rec date: January 15, 2014, Acc date: January 21, 2015, Pub date: January 27, 2015

Copyright: ( 2015 Hegazy RA. This is an open-access article distributed under the terms of the Creative Commons Attribution License, which permits unrestricted use, distribution, and reproduction in any medium, provided the original author and source are credited.

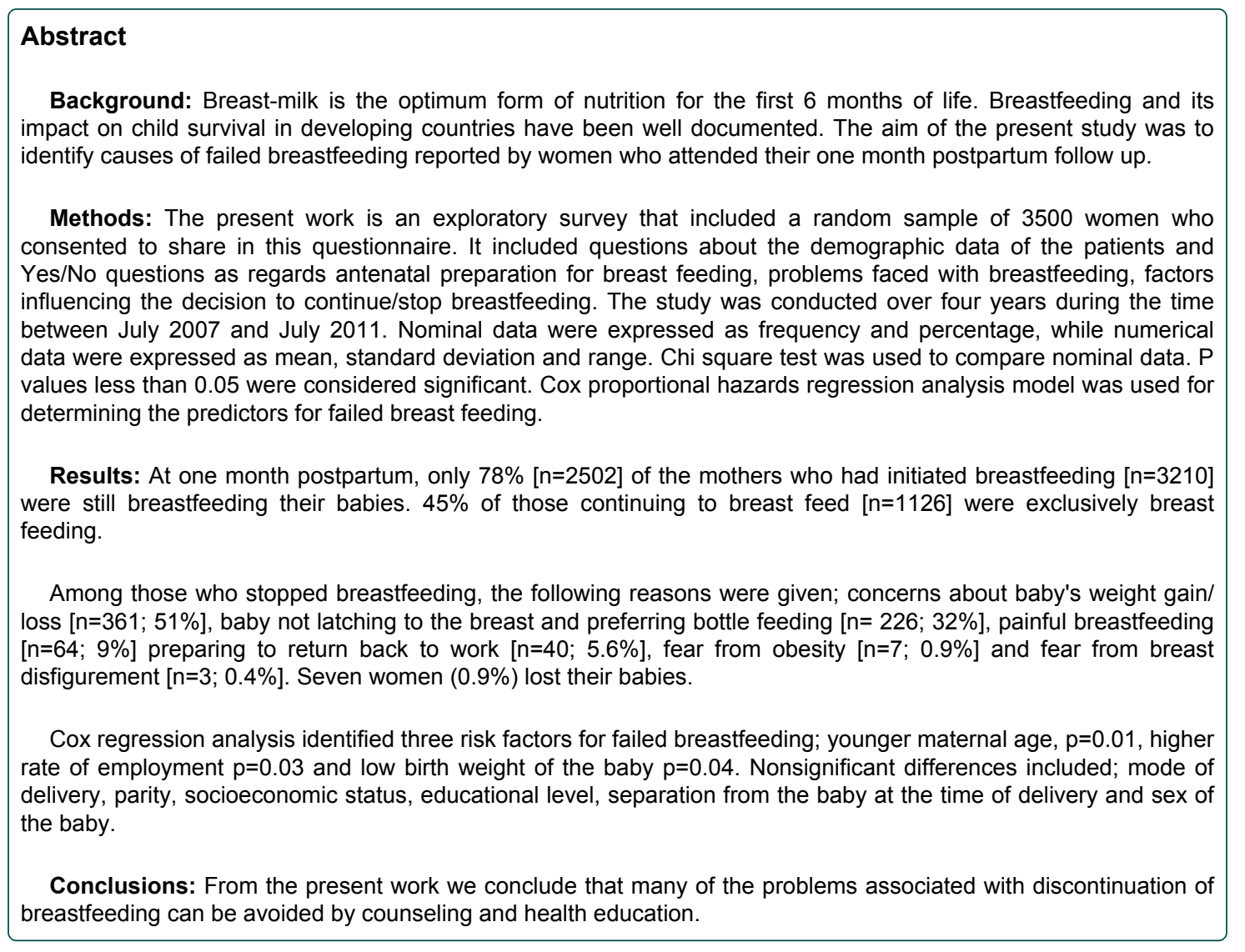

Keywords: Postpartum; Child survival; Breastfeeding

\section{Introduction}

Breast-milk is the optimum form of nutrition for the first 6 months of life [1].Breastfeeding and its impact on child survival in developing countries have been well documented. Governments are being urged to encourage breastfeeding through legislation and promotional campaigns. The success of promotional programs depends not only on the interventions themselves but on the acceptance and acquisition of the desired knowledge, skills and behaviors [2]. Each year more than 10 million children under the age of five years die, mainly from malnutrition among a short list of causes, and the majority live in low- income countries [3]. Although often an invisible phenomenon, malnutrition casts long shadows, affecting close to 800 million people, $20 \%$ of all people in the developing world [4]. The data obtained by the Egyptian Demographic Health Survey (EDHS) 2003 indicated that $16 \%$ of Egyptian under five children are stunted, $4 \%$ are wasted, and $8 \%$ are underweight [5].

In the National Population Council (NPC) study [6] conducted by choosing a nationally representative sample of adolescents (10-19 years old), $17 \%$ were stunted, $9 \%$ were thin, and $46.6 \%$ were anemic. Millennium Development Goal number 4 is to reduce child mortality by two thirds by 2015 [7]. Appropriate feeding practices are of fundamental importance for the survival, growth, development, health and nutrition of infants and young children. It is argued that 
promotion of exclusive breastfeeding is the most effective child health intervention currently feasible for implementation at population level in low-income countries $[8,9]$. Of the women who initiate breastfeeding, many cease in the first month following birth for reasons that might be avoided. More than half of mothers intend to breast-feed, yet many are unsuccessful at sustaining breast feeding beyond the first few weeks postpartum [10].

The aim of the present study was to describe breastfeeding difficulties reported by women who attended their one month postpartum follow up. Identifying their difficulties would help upgrade breastfeeding promotional programs through defining problems and high-risk groups and hence developing targeted approaches that would help promote breastfeeding.

\section{Methods}

The sample size was estimated to be 3500 with a confidence interval of $95 \%$, margin of error; $5 \%$. The present work is an exploratory survey that included 3500 women who consented to share in this questionnaire. Participants were recruited through systematic random sampling. After explaining the purpose of this study, the consenting women were given an Arabic questionnaire that was prepared based on electronic database searches, literature reviews and consultation with experts in the field. It included questions about the demographic data of the patients [age, parity, education level, employment] and Yes/No questions as regards antenatal preparation for breast feeding, problems faced with breastfeeding, factors influencing the decision to continue/stop breastfeeding. The study was conducted over four years during the time between July 2007 and July 2011. Women were equally recruited from postpartum follow up clinics at two university hospitals and two private hospitals, aiming to include all social sectors from the Egyptian community. After the study period, the relevant data was extracted from the questionnaire and analyzed using Microsoft Excel 2003 and SPSS version 14. Nominal data were expressed as frequency and percentage, while numerical data were expressed as mean, standard deviation and range. Chi square test was used to compare nominal data and relative risk $[R R]$ was used to determine the relation between patient factors. $\mathrm{P}$ values less than 0.05 were considered significant. Cox proportional hazards regression analysis model was used for determining the predictors for failed breast feeding. For the purpose of this study failed breast feeding was defined as complete cessation of breast feeding.

\section{Results}

\section{Demographic data}

The 3500 participants in this study had a mean age of $26 \pm 4.2$ years with a range of 19-34 years. 1785 [51\%] of the study group had received a higher education (completed further education beyond high school). 2380 [68\%] of the women were primigravidas, 790 [22.5\%] had one other baby, 280 [8\%] had two other children and 50 [1.4\%] had more. The vast majority were married while 25 [0.7\%] were divorced. 2200 [63\%] were employed and of those; 1510 [68.6\%] were planning to go back to work.

\section{Delivery data}

1820 [52\%] had given birth by cesarean section and 1680 [48\%] by normal vaginal delivery. 3020 [86\%] of the mothers gave birth to healthy full term babies that were roomed in with their mothers at the time of delivery. $98 \%$ of the interviewed mothers [ $n=3430]$ gave birth to single babies and $2 \% ; \mathrm{n}=70$ gave birth to twins.

490 [14\%] of the pregnancy outcomes required some form of assistance and were admitted to the nursery for reasons that included neonatal jaundice $[240 ; 48.9 \%]$, prematurity $[190 ; 38.7 \%]$, respiratory distress and others $[60 ; 12.2 \%]$. These babies were separated from their mothers for a period that ranged from two days to six weeks with a median of 7.3 days. However all mothers were encouraged to continue breast feeding if their babies' condition allowed it or otherwise, pump their breast milk and babies were given expressed breast milk during their period of stay at the nursery. None had contraindications to receive breast milk. Seven of the babies admitted to the NICU died.

\section{Breast feeding initiation}

3210 [91.7\%] of the interviewed mothers reported that they initiated breast feeding at the time of their delivery. Of the 290 mothers who didn't initiate breast feeding, 210 had previous failed breast feeding experiences, 50 women had retracted nipples and believed they were unable to breast feed and 30 women had previous breast surgeries and were informed that they would not be able to breast feed. One hundred and seventy women of those who didn't initiate breastfeeding reported to be smokers.

Those who didn't initiate breast feeding were significantly younger $[\mathrm{OR}=3.4,95 \%$ CI 1.12-10.72; $\mathrm{p}=0.01]$, showed significantly higher prevalence of smoking $[\mathrm{p}=0.02]$ and of delivery by cesarean section $[\mathrm{p}=0.02]$.

Mothers who gave birth by cesarean section initiated breast feeding had a significantly less initiation rates; $89 \%$ versus $94 \%$ in those who gave birth by vaginal delivery; $\mathrm{p}=0.02$. They also initiated breastfeeding significantly later [ $9.5 \pm 8$ hours] than those who gave birth by vaginal delivery [ $2.8 \pm 1.2$ hours; $\mathrm{p}=0.001]$.

2970 [84.8\%] of the study group reported that they were encouraged to practice nipple preparation during the last trimester and 1860 [53.1\%] reported to actually practice nipple stretch exercises and use nipple creams during the last trimester. Although initiation rates of breast feeding were comparable among women who practiced and those who didn't practice nipple preparation $[\mathrm{p}=0.23]$, the former group reported that they felt more confident about their ability to breast feed than the latter group. Again pain scores during breast feeding were not statistically different among both groups $[p=0.3]$.

Among the multiparous mothers, the previous breast feeding experience was identified as the single most important determining factor of their current view about breast feeding. Among the primiparous groups, concepts about breast feeding were derived from their obstetrician [46\%], experience of a close friend or relative [28\%], information read about breastfeeding, through media, books or internet [26\%].

\section{Breast feeding at one month}

At one month postpartum, only $78 \%$ [ $n=2502]$ of the mothers who had initiated breastfeeding [ $n=3210]$ were still breastfeeding their babies. $45 \%$ of those continuing to breast feed [ $n=1126]$ were exclusively breast feeding.

Among those who stopped breastfeeding, the following reasons were given; concerns about baby's weight gain/loss [ $\mathrm{n}=361 ; 51 \%]$, baby not latching to the breast and preferring bottle feeding [n=226;32\%], 
painful breastfeeding $[\mathrm{n}=64 ; 9 \%]$ preparing to return back to work $[\mathrm{n}=40 ; 5.6 \%]$, fear from obesity $[\mathrm{n}=7 ; 0.9 \%]$ and fear from breast disfigurement $[\mathrm{n}=3 ; 0.4 \%]$. Seven women $(0.9 \%)$ lost their babies.

Cox regression analysis identified three risk factors for failed breastfeeding

1. Younger maternal age; as mothers who didn't breastfeed were significantly younger with a mean age of 22.3 years versus a mean age of 26.3 years for those who breast fed $\mathrm{p}=0.01$. Mothers younger than the age of 25 years were more likely not to initiate breast feeding.

2. Higher rate of employment $\mathrm{p}=0.03$.

3. Low birth weight of the baby $\mathrm{p}=0.04$. Nonsignificant differences included; mode of delivery, parity, socioeconomic status, educational level, separation from the baby at the time of delivery and sex of the baby. Those who didn't initiate breastfeeding were excluded from the cox regression analysis.

$72 \%[n=510]$ of those who stopped breastfeeding believe that they might have continued to breast feed if they had received support and information from a health professional.

\section{Discussion}

The determinants of initiating breast-feeding vary among different populations, but knowledge of them is of fundamental importance for guiding programs to promote breast-feeding.

Among our study population, 3210 [91.7\%] initiated breast feeding. The main problems identified as hinders for initiation for breast feeding were young age, cesarean section and retracted nipples.

The role of cesarean section in delaying initiation of breast feeding has been previously studied. Cesarean delivery was defined as a risk factor for not initiating breast-feeding, for breast-feeding less than 1 month, and remained a significant hazard against breast-feeding duration [11]. It was also a significant barrier to the implementation of Baby Friendly Hospital Initiative Step 4 [12]. It is desirable to provide additional breast-feeding support during the early postpartum period to women who deliver via cesarean sections and these findings provide additional evidence in support of the avoidance of unnecessary obstetric interventions.

Fifty patients with retracted nipples $[17.2 \%$ of those who didn't initiate breast feeding] were found to lack confidence to initiate breast feeding. Nipple stretch exercises were not significantly associated with higher initiation rates or lower pain scores. However those practicing them felt more prepared for breast feeding. Previous studies have shown no basis for recommending the use of either Hoffman's nipple stretching exercises or breast shells as antenatal preparation for women with inverted and nonprotractile nipples who wish to breast feed [13]. Recommending nipple preparation with breast shells may sometimes reduce the chances of successful breast feeding, because it enforces the idea that there is a problem that might hinder breast feeding [14].

Although young maternal age was not reported in many studies to be related to initiation of breast feeding $[12,15,16]$, our results showed that initiation of breastfeeding were inversely correlated to maternal age. Mothers younger than the age of 25 years were more likely not to initiate breast feeding; hence this age group represents a critical group that might need particular support from breast feeding counselors.
However, the role of age needs to be analyzed after correction for confounding factors.

The majority of those who didn't initiate breast feeding were smokers which is in agreement with previous reports that current parental smoking habits only affected breast-feeding initiation, though unrelated to breast-feeding duration [11].

Most of the problems reported by mothers who discontinued breast feeding were similar to those reported in previous surveys $[9,15,17]$ and many were found to be preventable. Women need more accurate information about the process of breastfeeding. Nurses should avoid teaching breastfeeding practices that can confuse infants and sabotage mothers and should work toward providing sensitive care that respects personal boundaries of mothers. Additionally, provisions need to be made for continued support and consultation after the mother's discharge from the hospital or birthing center. Many of the problems interfering with breast feeding can be avoided by structured advice and guidance from a health professional. Interventional studies comparing rates before and after breastfeeding counseling will further confirm those beliefs [18].

Maternal employment has long been viewed as a hindrance to continued breast feeding [19]. In the present work it presented an important factor contributing to stopping breast feeding, because of long hours of separation from the baby, difficulty in breast pumping and introduction of artificial feeding. Given the importance of breast feeding, collaborated efforts are needed to solve this problem. Making work space available for breast feeding, increasing the paid leaves to six months, encouraging breast milk pumping and storage are some of the proposed solutions.

Low birth weight is a risk factor for discontinued breast feeding. Periods of separation due to admission in NICU, stress for catch up growth and promotion of high calorie infant formula are all related to the problem.

\section{Conclusions}

From the present work we conclude that many of the problems associated with discontinuation of breastfeeding can be avoided by counseling.

Given the extreme value of breastfeeding all efforts expended in increasing its rate are worth it. Further larger interventional studies are needed to validate the influence of counseling and education.

\section{References}

1. Berridge K, McFadden K, Abayomi J, Topping J (2005) Views of breastfeeding difficulties among drop-in-clinic attendees. Matern Child Nutr 1: 250-262.

2. Wilmoth TA, Elder JP (1995) An assessment of research on breastfeeding promotion strategies in developing countries. Soc Sci Med 41: 579-594.

3. Ibrahim B, Sallam S, El Tawila S, El Gibaly O, El Sahn F, et al. (1999) Transitions to adulthood, a national survey of Egyptian adolescents.

4. UNICEF: The United Nations Children's Fund. UNICEF's priorities for children 2002-2005.

5. El-Zanaty F, Way AA (2004) Egypt interim demographic and health survey 2003. Ministry of Health and Population (Egypt), National Population Council (Egypt)(Cairo) and ORC Macro.

6. Black RE, Morris SS, Bryce J (2003) Where and why are 10 million children dying every year? Lancet 361: 2226-2234.

7. United Nations Statistics Division: Progress towards the Millennium Development Goals, 1990-2005. 
Citation: Hegazy RA, Abdelaziz SB, Fahmy AA, Shaeer EK (2015) Failed Breast Feeding among Egyptian Women at One Month Postpartum: A Cross-Sectional Community Based Study. Clinics Mother Child Health 12: 170. doi:10.4172/2090-7214.1000170

Page 4 of 4

8. Jones G, Steketee RW, Black RE, Bhutta ZA, Morris SS (2003) How many child deaths can we prevent this year? Lancet 362: 65-71.

9. Fjeld E, Siziya S, Katepa-Bwalya M, Kankasa C, Moland KM, et al. (2008) No sister, the breast alone is not enough for my baby' a qualitative assessment of potentials and barriers in the promotion of exclusive breastfeeding in southern Zambia. Int Breastfeed J 3: 26.

10. Bedinghaus JM, Melnikow J (1992) Promoting successful breast-feeding skills. Am Fam Physician 45: 1309-1318.

11. Leung GM, Lam TH, Ho LM (2002) Breast-feeding and its relation to smoking and mode of delivery. Obstet Gynecol 99: 785-94.

12. Rowe-Murray HJ, Fisher JR (2002) Baby friendly hospital practices: cesarean section is a persistent barrier to early initiation of breastfeeding. Birth 29: 124-131.

13. (1994) The MAIN Trial Collaborative Group. Preparing for breast feeding: treatment of inverted and non-protractile nipples in pregnancy. Midwifery 10: 200-214.

14. Alexander JM, Grant AM, Campbell MJ (1992) Randomised controlled trial of breast shells and Hoffman's exercises for inverted and nonprotractile nipples. BMJ 304: 1030-1032.
15. Bautista LE (1997) Factors associated with the initiation of breast feeding by women in the Dominican Republic Rev Panam Salud Publica 1: 200-207.

16. Kulsoom U, Saeed A (1997) Breast feeding practices and beliefs about weaning among mothers of infants aged 0-12 months. J Pak Med Assoc 47: 54-60.

17. Bedinghaus JM, Melnikow J (1992) Promoting successful breast-feeding skills. Am Fam Physician 45: 1309-1318.

18. Mozingo JN, Davis MW, Droppleman PG, Merideth A (2000) "It wasn't working. "Women's experiences with short-term breastfeeding. Am J Matern Child Nurs 25: 120-126.

19. Noble S (2001) ALSPAC Study Team. Avon Longitudinal Study of Pregnancy and Childhood. Maternal employment and the initiation of breastfeeding. Acta Paediatr. 90: 423-428. 